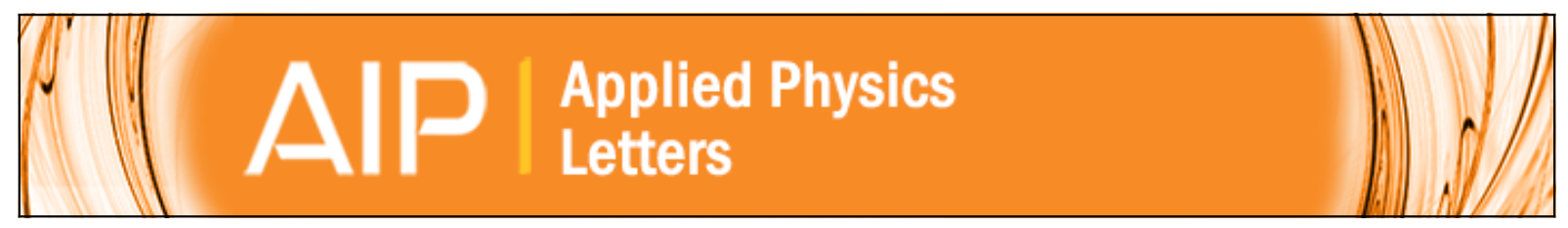

\title{
Characterization of energy trapping in a bulk acoustic wave resonator
}

Kimmo Kokkonen, Johanna Meltaus, Tuomas Pensala, and Matti Kaivola

Citation: Applied Physics Letters 97, 233507 (2010); doi: 10.1063/1.3521263

View online: http://dx.doi.org/10.1063/1.3521263

View Table of Contents: http://scitation.aip.org/content/aip/journal/apl/97/23?ver=pdfcov

Published by the AIP Publishing

\section{Articles you may be interested in}

Intrinsically switchable thin film bulk acoustic wave resonators

Appl. Phys. Lett. 104, 222905 (2014); 10.1063/1.4881141

Ferroelectric film bulk acoustic wave resonators for liquid viscosity sensing

J. Appl. Phys. 114, 084106 (2013); 10.1063/1.4819327

Extraction of second order piezoelectric parameters in bulk acoustic wave resonators

Appl. Phys. Lett. 100, 232901 (2012); 10.1063/1.4725503

Real-time imaging of acoustic waves on a bulk acoustic resonator

Appl. Phys. Lett. 93, 261101 (2008); 10.1063/1.3053074

Legendre polynomial modeling of composite bulk acoustic wave resonators

J. Appl. Phys. 104, 014508 (2008); 10.1063/1.2953096
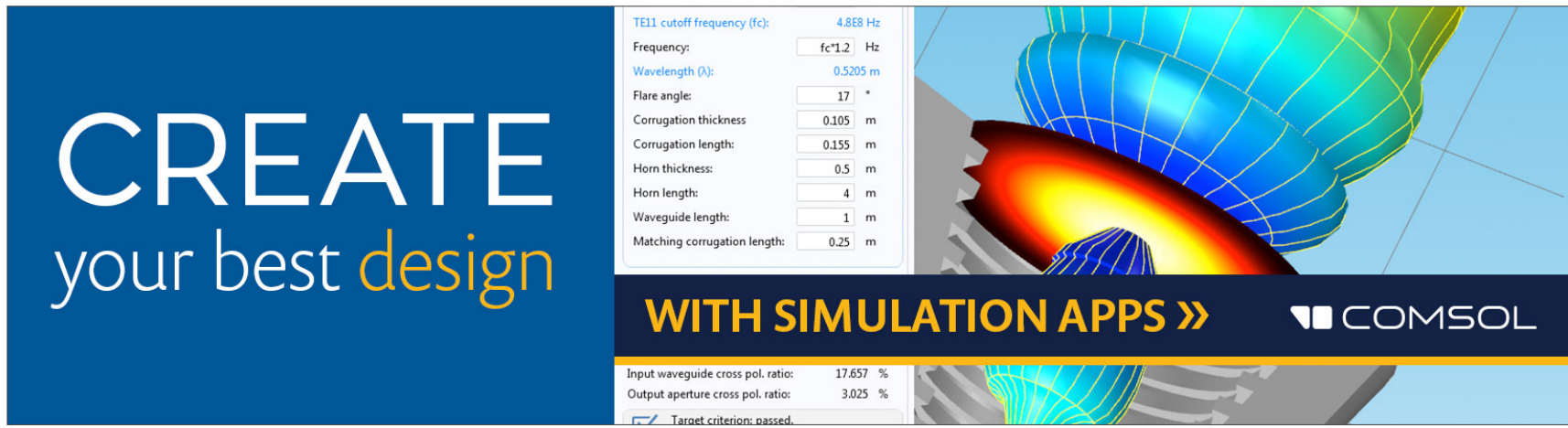


\title{
Characterization of energy trapping in a bulk acoustic wave resonator
}

\author{
Kimmo Kokkonen, ${ }^{1, a)}$ Johanna Meltaus, ${ }^{2}$ Tuomas Pensala, ${ }^{2}$ and Matti Kaivola ${ }^{1}$ \\ ${ }_{1}^{1}$ Department of Applied Physics, Aalto University, Espoo 02150, Finland \\ ${ }^{2}$ VTT Technical Research Centre of Finland, P.O. Box 1000, 02044 VTT, Finland
}

(Received 14 July 2010; accepted 6 November 2010; published online 8 December 2010)

\begin{abstract}
Acoustic wave fields both within the active electrode area of a solidly mounted $1.8 \mathrm{GHz}$ bulk acoustic wave resonator, and around it in the surrounding region, are measured using a heterodyne laser interferometer. Plate-wave dispersion diagrams for both regions are extracted from the measurement data. The experimental dispersion data reveal the cutoff frequencies of the acoustic vibration modes in the region surrounding the resonator, and, therefore, the energy trapping range of the resonator can readily be determined. The measured dispersion properties of the surrounding region, together with the abruptly diminishing amplitude of the dispersion curves in the resonator, signal the onset of acoustic leakage from the resonator. This information is important for verifying and further developing the simulation tools used for the design of the resonators. Experimental wave field images, dispersion diagrams for both regions, and the threshold for energy leakage are discussed. (C) 2010 American Institute of Physics. [doi:10.1063/1.3521263]
\end{abstract}

Thin-film bulk acoustic wave (BAW) resonators are widely used in modern mobile communication systems. Their high Q-value at gigahertz frequencies, bandwidth, temperature stability, and power durability combined with a small size make possible the realization of filters fulfilling demanding specifications. To obtain a low-loss BAW resonator, the acoustic energy must be trapped within the resonator both in the vertical and in the lateral directions. The former is realized with an acoustic Bragg reflector or an air gap that isolates the resonator from the substrate. For the lateral energy trapping, the plate-wave propagation characteristics, i.e., the wave dispersion, should be such that there are no propagating wave modes in the region surrounding the resonator (outside region) at or near the operation frequency of the resonator. The dispersion behavior of a BAW device is determined by, and can be tailored with, the design of the thin-film layer stack. Often the dispersion properties of actual resonators differ from simulations due to, for example, inaccurate material parameters. It is therefore important to be able to measure the dispersion properties.

Optical probing is a powerful characterization method for studying mechanical wave fields in microacoustic components, such as BAW resonators. ${ }^{1-7}$ Laser interferometry is a noncontact optical method that enables direct measurement of wave fields in an acoustic component. It has been also used to characterize the plate-wave dispersion properties in thin-film BAW resonators (see, e.g., Refs. 8-10). These measurements have been limited to characterizing the dispersion in the electrode area of the component, thereby yielding only partial information of the acoustic behavior, critical for the component operation. As an example, in order to study energy trapping in BAW resonators, one must be able to extract dispersion curves not only within the active area of a resonator but also in other regions, especially in the region surrounding the electrode. Since the resonator is excited by a voltage applied at the electrode, the measurements outside the electrode region call for a sensitive measurement setup because the vibration fields in the outside area are only due

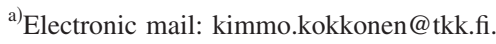

to leakage of acoustic energy from the electrode to its surroundings. Furthermore, to be able to extract the outside region's dispersion properties from the measurement data, one needs to apply specific data processing methods.
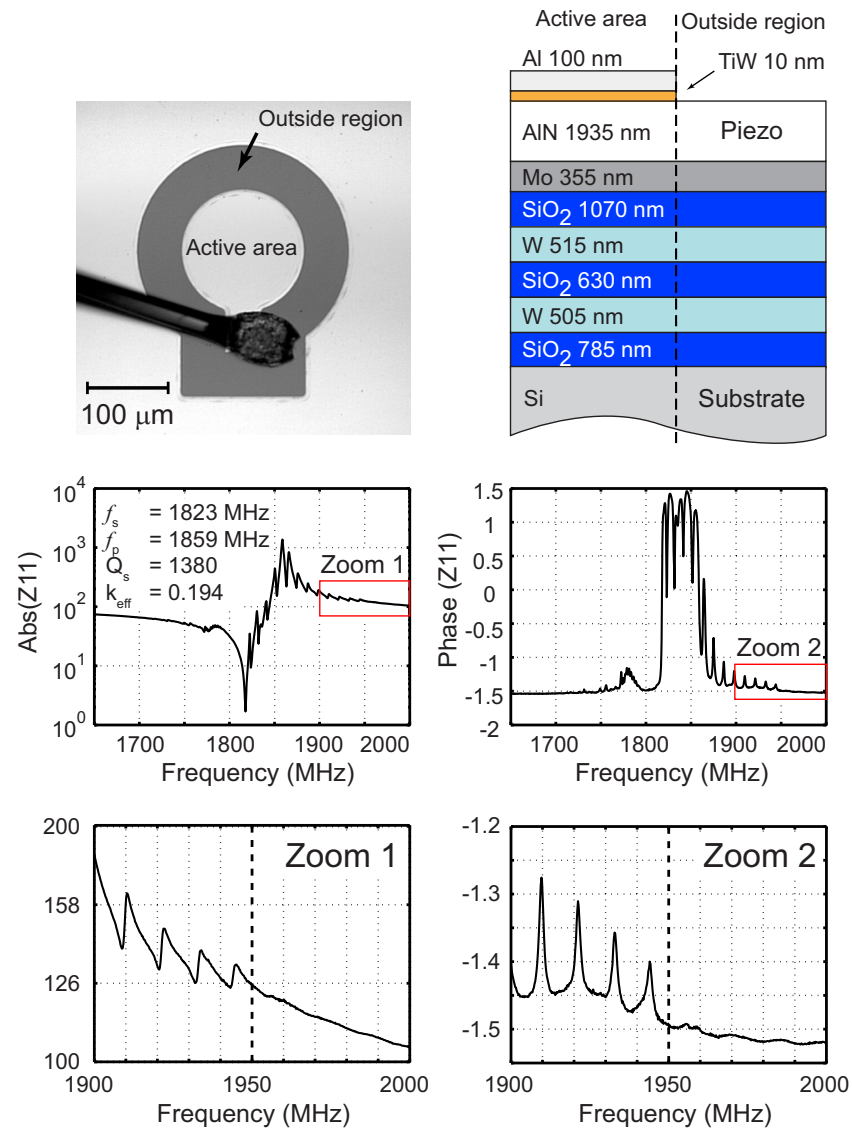

FIG. 1. (Color online) Top-left: Microscope image of the sample wirebonded to a jig for interferometric measurements. Top-right: Schematic view of the layer stack of the sample, with the regions defined. Electrical measurements: Impedance and phase response obtained with wafer-level measurement. Main figures of merit are provided as an inset. The electrical response features spurious resonances. A zoomed view to the electrical response is provided to show the sudden disappearance of the spurious responses at around $1950 \mathrm{MHz}$. 


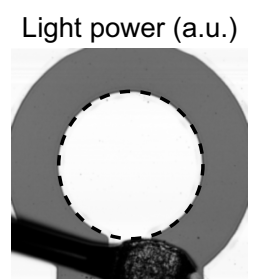

$1815 \mathrm{MHz}$

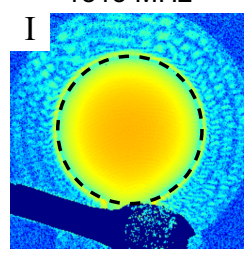

$1947 \mathrm{MHz}$

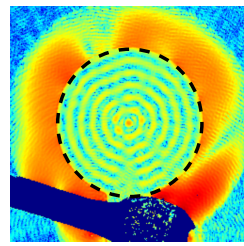

$1769 \mathrm{MHz}$

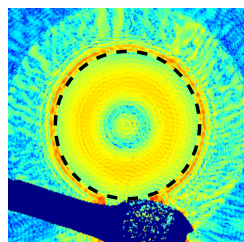

$1823 \mathrm{MHz}$

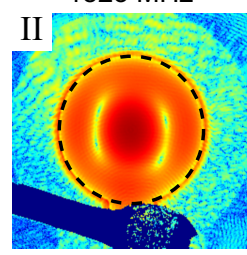

$1949 \mathrm{MHz}$

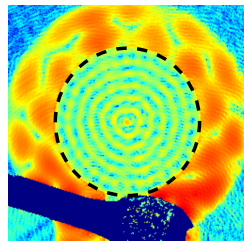

$1774 \mathrm{MHz}$

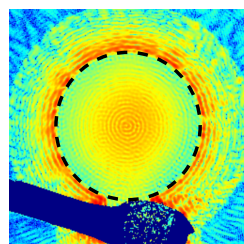

$1841.5 \mathrm{MHz}$

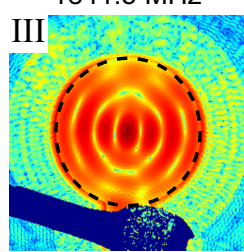

$1959 \mathrm{MHz}$

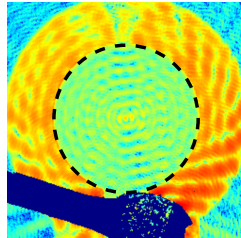

$1776 \mathrm{MHz}$

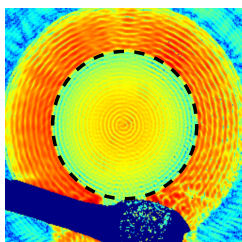

$1874.5 \mathrm{MHz}$

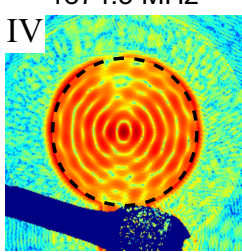

$1970 \mathrm{MHz}$

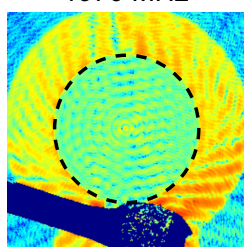

$1778 \mathrm{MHz}$

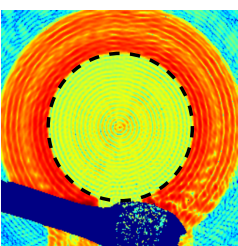

$1933 \mathrm{MHz}$

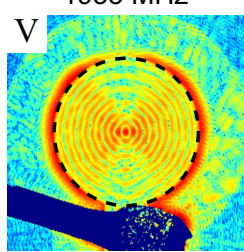

$1985 \mathrm{MHz}$

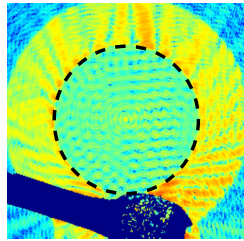

(a)

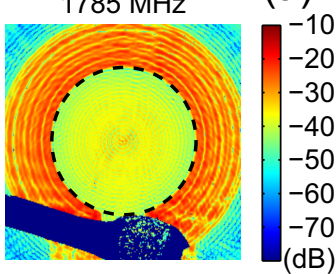

$1944 \mathrm{MHz}$

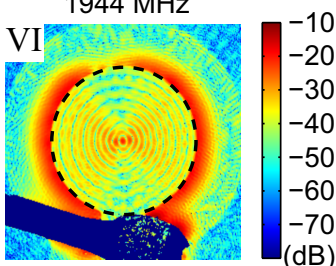

$2000 \mathrm{MHz}$

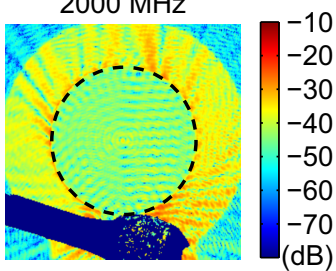

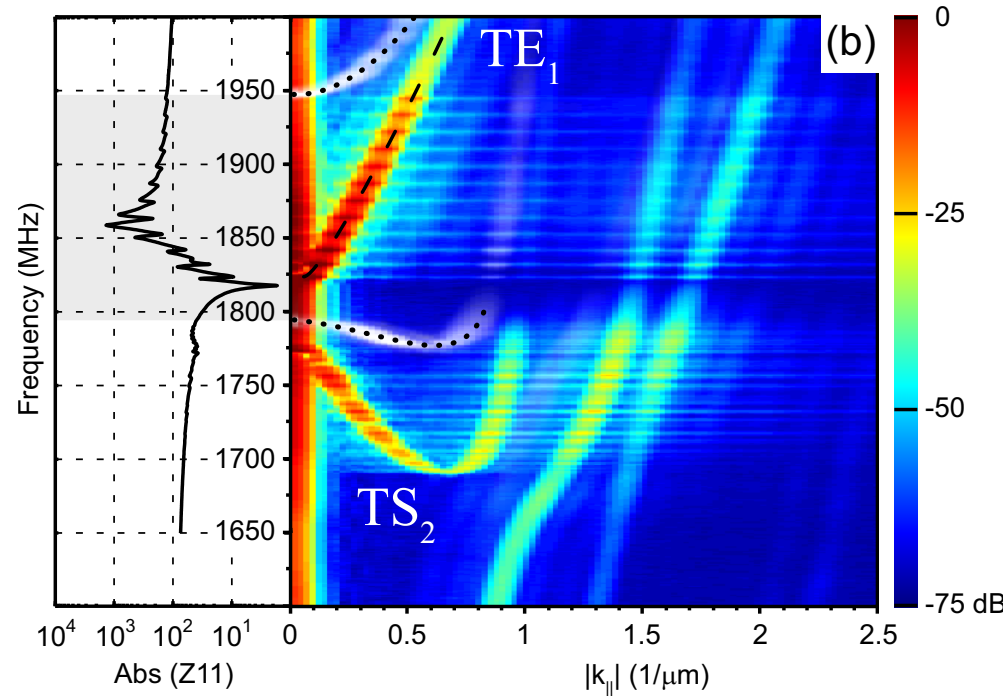

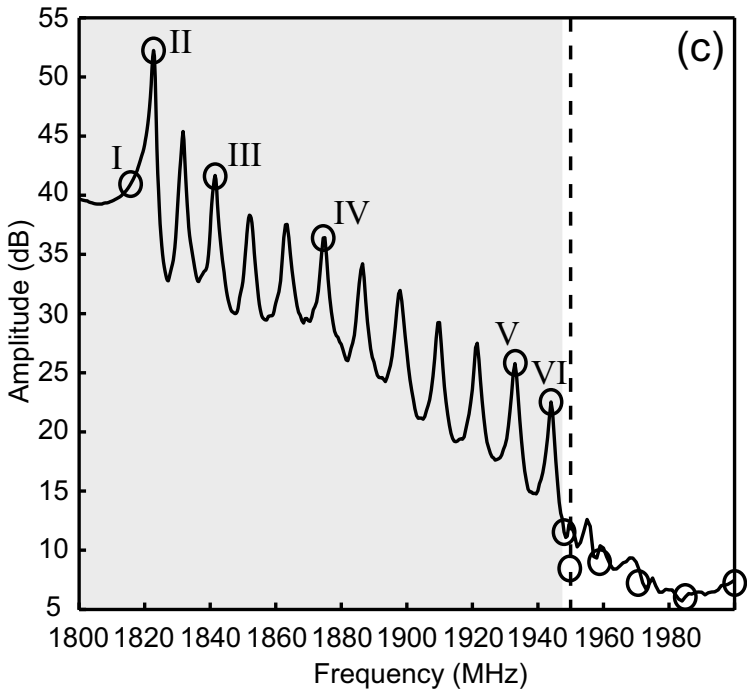

FIG. 2. (Color) (a) Top: Light power image of the scan area and five vibration amplitude images at selected frequencies, at which $\mathrm{TS}_{2}$ modes in the resonator and the outside region are active. The edge of the resonator is marked with a black dashed line. Middle: Six vibration amplitude images selected to show the resonator behavior when the outside region does not support propagating waves. Mode trapping to the active area is evident, with an evanescent tail increasing

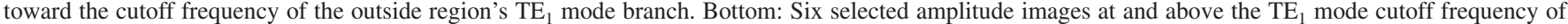
the outside region with energy coupling from the resonator to its surroundings clearly visible. (b) Dispersion diagram for both areas. The dispersion diagram from the active area of the resonator is plotted with colors ranging from deep blue to red. The dispersion diagram obtained from the outside area is superposed by using shades of gray, and the mode branches are indicated with a dotted line. The electrical impedance response is presented on the left hand side for reference. (c) The amplitude along the $\mathrm{TE}_{1}$ mode branch is presented as a function of frequency with circles marking the frequencies of the 12 wave field images presented on the middle and bottom rows in (a). The dashed line at $1950 \mathrm{MHz}$ is for reference. The energy trapping range, determined from the dispersion measurements, is denoted by a gray shaded area in the impedance response curve in (b) and in the amplitude graph (c).

In this letter, we present a method to obtain dispersion data both from the resonator and its surrounding region using laser-interferometric measurements and data processing. Both sets of dispersion diagrams can be obtained with a single measurement. The dispersion data of the active and outside regions allow more complete characterization of the acoustic behavior of the BAW component. As an example, one can directly determine the frequency range of lateral trapping of acoustic energy.

The sample resonator consists of five alternating layers of $\mathrm{W}$ and $\mathrm{SiO}_{2}$, which form the acoustic reflector and a piezoelectric AlN layer between a Mo bottom electrode and an
Al top electrode. A photograph of the sample is presented in Fig. 1, with a schematic picture of the thin-film stack and the input impedance $Z_{11}$ extracted from wafer-level vector network analyzer measurements. The electric frequency response shows a resonance at $1823 \mathrm{MHz}$ and an antiresonance at $1859 \mathrm{MHz}$. Sharp spurious oscillations caused by laterally standing plate-waves with a nonzero lateral wavenumber $k_{\|}$ are superposed onto the resonance-antiresonance curve. These spurious resonances can be suppressed, for example, with a boundary structure. ${ }^{11}$ In this sample, however, we did not suppress them in order to be able to study their 
properties $^{12}$ and their connection to the energy trapping condition.

A scanning heterodyne laser interferometer ${ }^{5}$ was used to acquire the phase and absolute amplitude of the surface vibration field. The measurement area of $250 \times 250 \mu \mathrm{m}^{2}$ contains the circular active area (electrode) of the resonator and some of the nonmetallized outer region. The measured frequency range was 1600-2000 MHz, lateral scan step was $1 \mu \mathrm{m}$, and the resonator was driven from a $50 \Omega$ transmission line with a nominal input power of $5 \mathrm{dBm}$.

Measured wave fields are presented in Fig. 2(a). The amplitude fields on the first and third rows indicate that the outside region is strongly activated at frequencies around 1778 and $1947 \mathrm{MHz}$. In order to separately extract the dispersion of the active and outside areas, the measurement data were windowed before applying Fourier transform. To calculate the dispersion of the active area, we exclude the outside region from the analysis, and conversely, to calculate the dispersion of the outside region, the active region is excluded, leaving a doughnut-shaped area for the analysis. Resulting dispersion diagrams for both areas are presented in Fig. 2(b). The dispersion of the active area is shown with a colormap ranging from dark blue to red, whereas the dispersion of the outside region is superposed with shades of gray. The $\mathrm{TE}_{1}$ and $\mathrm{TS}_{2}$ mode branches of the outside region are also indicated with a dotted line.

From the electrical measurements of Fig. 1, we can see that at around $1950 \mathrm{MHz}$ the spurious resonances disappear and the electrical response becomes smooth. From the electrical measurements alone, however, it is difficult to determine the cause and the exact threshold frequency of that behavior. From the dispersion diagram of Fig. 2(b), on the other hand, one can readily see that the $\mathrm{TE}_{1}$ mode of the outside region emerges at around $1947 \mathrm{MHz}$. In the wave field images of Fig. 2(a), this is seen as the activation of the outside area. Above this frequency, acoustic energy in the resonator couples to propagating waves in the surrounding region. To further illustrate this behavior, we have plotted the amplitude of the electrode area's $\mathrm{TE}_{1}$ mode dispersion as a function of frequency in Fig. 2(c). The eigenresonances vanish after the cutoff frequency of the outside region.

We have shown a method to extract dispersion characteristics of the nonactive area of a BAW resonator, and the method is illustrated by directly determining the lateral energy trapping range of a resonator. Both dispersion sets (active and outside areas) can be obtained with the same scan. The two regions of interest are separated using data windowing. The measured acoustic data correlate with the effects observed in electric measurements and directly verifies that the reason for the disappearance of spurious resonances from the electric response is the onset of lateral acoustic leakage, something which cannot be deduced from the electric measurements or from the active area dispersion data only.

K.K. thanks the Finnish Cultural Foundation and the Nokia Foundation for scholarships.

${ }^{1}$ J. V. Knuuttila, P. T. Tikka, and M. M. Salomaa, Opt. Lett. 25, 613 (2000).

${ }^{2}$ J. E. Graebner, B. P. Barber, P. L. Gammel, D. S. Greywall, and S. Gopani, Appl. Phys. Lett. 78, 159 (2001).

${ }^{3}$ G. G. Fattinger and P. T. Tikka, Appl. Phys. Lett. 79, 290 (2001).

${ }^{4}$ K. L. Telschow, V. A. Deason, D. L. Cottle, and J. D. Larson III, IEEE Trans. Ultrason. Ferroelectr. Freq. Control 50, 1279-1285 (2003).

${ }^{5}$ K. Kokkonen and M. Kaivola, Appl. Phys. Lett. 92, 063502 (2008).

${ }^{6}$ T. Fujikura, O. Matsuda, D. M. Profunser, O. B. Wright, J. Masson, and S. Ballandras, Appl. Phys. Lett. 93, 261101 (2008).

${ }^{7}$ K.-Y. Hashimoto, K. Kashiwa, T. Omori, M. Yamaguchi, O. Takano, S. Meguro, and K. Akahane, Proceedings of the IEEE MTT-S International Microwave Symposium, 2008, pp. 851-854.

${ }^{8}$ J. E. Graebner, H. F. Safar, B. Barber, P. L. Gammel, J. Herbsommer, L. A. Fetter, J. Pastalan, H. A. Huggins, and R. E. Miller, Proceedings of the IEEE Ultrasonics Symposium, 2000, Vol. 1, pp. 635-638.

${ }^{9}$ T. Makkonen, T. Pensala, J. Vartiainen, J. V. Knuuttila, J. Kaitila, and M. M. Salomaa, IEEE Trans. Ultrason. Ferroelectr. Freq. Control 51, 42 (2004).

${ }^{10} \mathrm{~K}$. L. Telschow and J. D. Larson, Proceedings of the IEEE Ultrasonics Symposium, 2006, pp. 448-451.

${ }^{11}$ J. Kaitila, M. Ylilammi, J. Ellä, and R. Aigner, Proceedings of the IEEE Ultrasonics Symposium, 2003, pp. 84-87.

${ }^{12}$ K. Kokkonen, T. Pensala, J. Meltaus, and M. Kaivola, Appl. Phys. Lett. 96, $173502(2010)$. 